\title{
CHARACTERISTICA UNIVERSALIS
}

\section{PREAMBLE}

Our task will be to construct portions of a directly depicting language which will enable us to represent the most general structures of reality. We shall draw not on standard logical treatments of the contents of epistemic states as these are customarily conceived in terms of propositions. Rather, we shall turn to a no less venerable but nowadays somewhat neglected tradition of formal ontology, in which not sentences or propositions, but maps, diagrams or pictures, shall serve as the constituents of our mirror of reality.

The construction of a directly depicting language we conceive as being in a certain sense an experimental matter. One can, as Peirce remarked, 'make exact experiments upon uniform diagrams', and in formal ontology as we conceive it operations upon diagrams will 'take the place of the experiments upon real things that one performs in chemical and physical research.' ${ }^{1}$ The chemist is of course grappling in his experiments with the very bits of reality whose properties he is concerned to establish. Here, in contrast, we shall be experimenting at one remove, with diagrams of reality. The contrast is not so great as it might seem, however. For it is not, in fact, the particular samples in which the chemist is interested; rather (in Scotist vein):

the object of the chemist's research, that upon which he experiments, and to which the question he puts to Nature relates, is the Molecular Structure, which in all his samples has as complete an identity as it is in the nature of Molecular Structure ever to possess.

And now, as Peirce once more rightly insists, it is not otherwise with experiments made upon diagrams. The latter are 'questions put to the 
Nature of the relations concerned' - precisely in virtue of the fact that we are here experimenting with diagrams which are to enjoy the property that the forms of relations exemplified in reality will be the very same as the forms of relations in the diagrams themselves.

A similar idea is of course present also in Wittgenstein. As the Tractatus has it: 'What constitutes a picture is that its elements are related to one another in a determinate way.' (2.41) Indeed: 'There must be something identical in a picture and what it depicts, to enable the one to be a picture of the other at all.' (2.16) Wittgenstein's 'pictorial form', then, is Peirce's 'form of a relation', and our task here will be one of taking further the idea of a universal characteristic which both philosophers shared.

\section{FROM LEIBNIZ TO FREGE}

The project of such a characteristic had of course been envisaged by Leibniz, and the idea is present already in Descartes and Jungius and in others before them. ${ }^{2}$ In each case we have the idea that it is possible to isolate a relatively small number of basic units and of structure-building principles governing the combination of such units in a way which will allow, by more or less mechanical means, the felicitous representation of all the concepts, truths, thoughts, or structures, pertaining to a given sphere. The crucial idea here is that of compositionality. Already Jungius saw the mathematical method for the study of nature as valid precisely because

nature does not act the way the Chinese write, but like other peoples, i.e., with an alphabet ... through combinations, complications, and replications of a few hypotheses, laws, or principles $^{3}$

In the work of Descartes, Jungius and Leibniz, however, two distinct ideas are run together: the idea of the characteristic as a perspicuous representation of relations among concepts, and the idea of the characteristic as a mirror of reality. Only in the brief realist interregnum around the turn of the present century, and especially in the work of Brentano and his followers, in the Tractatus, and to some extent also in Peirce, did the second of these two ideas, the idea of an ontological characteristic, come into its own.

Brentano himself applies this idea to the specific field of psychology. Brentanian descriptive psychology is accordingly an example of a characteristica specialis, a directly depicting language restricted to some 
specific sphere. That he is seeking an ontological characteristic in our sense is nonetheless clear. He describes the discipline of descriptive psychology as having been conceived as an instrument that would

display all the ultimate psychic components, from whose combination one with another the totality of psychic phenomena would result, just as the totality of words is yielded by the letters of the alphabet. ${ }^{4}$

The project of a characteristica universalis, of a directly depicting language that would apply to all spheres of reality without restriction, is sketched by Husserl in the Logical Investigations - a work which can in many respects be conceived as an extrapolation of Brentanian ideas in the direction of a completely general formal ontology. Husserl provides however no more than philosophical preliminaries to the working out of the formal details of a characteristic. The first sophisticated moves in this formal direction after Peirce are provided, rather, in the work of Frege, who had the key idea underlying the project of a directly depicting language that the syntactic relations of expressions in such a language should mirror exactly corresponding ontological relations among the entities depicted. Moreover, Frege first showed the possibility of a new sort of syntactic precision and determinacy in this respect - specifying for the first time a language with a definite notion of well-formedness. The expressions of Frege's system are, familiarly, built up via the nexus of function and argument: one expression is said to 'saturate' another (incomplete) expression, as when we move from 'the square root of' to 'the square root of 2'. (The chemical connotations of Frege's terminology of 'saturation' here are not accidental.)

In some of his earlier writings, now, Frege suggests that the complex expressions of his system should map corresponding structures among saturated and unsaturated entities in the world, in such a way that, when a function is saturated by an argument, then what results is a whole of which the function is a part. (Wittgenstein, too, seems to presuppose a similar idea e.g. at 5.47, where he suggests that a thing's being composite involves in every case function and argument.) Certainly it may be argued that mereological relations are preserved in this way when functional expressions are saturated by other expressions of an appropriate type. The expression 'father of' is in a certain sense part of the more complex expression 'father of John'. A moment's reflection makes it clear, however, that such mereological relations on the syntactic level cannot, on the Fregean approach, mirror corresponding relations among the 
objects in the world - John himself is not a part of his father, any more than Denmark, say, is a part of Copenhagen. Thus as Dummett points out, Frege quickly saw that it had been wrong for him ever to have suggested that a parallelism of the given sort can be maintained. ${ }^{5}$ The most that can be affirmed is that the unsaturatedness of a functional expression mirrors a corresponding unsaturatedness in the function for which it stands.

Frege does hold on to the idea of a characteristic language in certain respects, however. Above all, the absence of semantics in Frege's logic puts him firmly within the tradition of characteristic ontology as here conceived. A truly adequate directly depicting language does not need a semantics, for the relation of language and world is here able to take care of itself. Frege's clear departure from the characteristic tradition is seen, however, in his insistence that all expressions of his system, whether saturated or unsaturated, are referential - so that he spurns the admission of empty complex names (names which would refer to nothing at all because the corresponding objects do not stand to each other in the way they are represented as standing). Frege's work is in this respect similar to that of Meinong, though the similarity is masked by Frege's tendency to identify the referents of names that would intuitively count as referring to what is non-existent - names like 'the grandmother of Denmark' - with some arbitrarily chosen dummy such as the False or the number 0. For other reasons, too, the names of Frege's language (and above all most names of the True and the False), do not mirror the structures of what they denote or represent. At best we might say that they mirror the structure of a certain method for fixing or identifying their respective denotata.

\section{DIRECTLY DEPICTING DIAGRAMS VS. EXISTENTIAL GRAPHS}

The failure of Frege's system as the basis of a characteristic flows, we might say, from the fact that he confused grammar and ontology. This is because Frege allowed intuitions deriving from investigations of grammatical well-formedness to determine his account of the structures of the objects depicted by the diagrams of his system. (Meinong allowed his ontology to be determined in a similar way by psychological considerations.) In a properly constructed characteristic language, in contrast, the relation of determination will flow in the opposite direction: the structures 
of diagrams will as far as possible be dictated by the structures in reality they are designed to represent. In particular, the directly depicting language to be constructed in what follows will hold as doggedly as possible to the idea that the part-whole relations among its constituent expressions must map exactly corresponding relations among the pictured objects. Thus we are interested, as Peirce, again, expressed it:

in constructing an icon or diagram the relations of whose parts shall present a complete analogy with those of the parts of the object of reasoning, of experimenting upon this image in the imagination, and of observing the result so as to discover unnoticed and hidden relations among the parts. (3.363)

Peirce was however interested above all in constructing diagrammatic languages for purposes of logic (though 'logic', for him, as for Frege, overlapped to some extent with what is here called 'formal ontology'). Peirce's existential graphs were designed to 'render literally visible before one's very eye the operation of thinking in actu.' (4.6) ${ }^{6}$ Here, in contrast, we are interested in the idea of constructing a diagrammatic language for purposes strictly ontological. Like Peirce we shall employ a modification of Euler's diagrams to this effect. ${ }^{7}$ Peirce saw it as a defect of such diagrams as originally conceived that they relate essentially to dichotomous relations among what is general, i.e. to relations among the ontological correlates of common names of the sort that are at issue for example in classical Aristotelian syllogistic. Thus the inclusion of one ovoid $\alpha$ inside another $\beta$ might indicate that the concept $\alpha$ is subordinate to the concept $\beta$ as red is subordinate to colour. Such diagrams operate entirely in the sphere of what is general. Hence they cannot be used to affirm the existence of any individual satisfying a given description. This may suffice for the purposes of the logician. The formal ontologist, however, is concerned with the depiction of what actually exists, and since he is concerned not merely with what is general but also with what is particular, his diagrammatic language must embrace also what we might call indexical or 'proper' diagrams, which will function in much the way that proper names function in ordinary language.

Our diagrams will in fact almost all of them incorporate proper names in the ordinary sense as constituent parts. It is from these that they will inherit their primary relation (be anchored) to reality. It will simply be assumed in what follows that such ordinary proper names refer; we shall not ask in virtue of what they refer, though we note that this would be a proper and pressing question for another occasion. We shall say that a 
diagram is true if that which it sets out to depict exists in reality. As 'clementary diagrams' we shall allow proper names standing alone; and it will turn out that, because empty proper names are not admitted, every elementary diagram of this sort is true. A complex diagram is true if and only if the structural relations between the names (and other bits and pieces in the diagram) map structural relations among the corresponding objects in the world. Otherwise it is false. All of this should, of course, be perfectly lamiliar. ${ }^{8}$ Our diagrams will furthermore be synchronous only. Thus by 'the world' or 'reality' we shall mean reality as it is now, at the present time. The treatment of time and change from the standpoint of a universal characteristic we postpone for the future.

\section{SOME CONDITIONS ON A DIRECTLY \\ DEPICTING LANGUAGE}

It is above all in the ideal language philosophy defended by Wittgenstein in the Tractatus, by Gustav Bergmann and his followers, and by Cocchiarella and others in the present day, that the project of a characteristica universalis has received its most sophisticated modern expression. Even these thinkers, however, too often run together ontological and logical concerns. Most importantly, they identify logical and ontological simplicity and complexity, and thereby also they assume that logical atomism implies one or other form of ontological atomism. Hence the project of a directly depicting 'ideal' language has come to be associated with atomistic doctrines of one or other sort as concerns the objects which such a language might depict. Consider, for example, Russell's atomism of thises and thats, Bergmann's doctrine of bare particulars, or Grossmann's modification of this doctrine in his The Categorial Structure of the World. Here, in contrast, the idea of a directly depicting language built up out of proper names in something like the Tractarian sense will be combined with a view of such proper names as referring to common-or-garden substances like Chisholm or this desk, and to the various different sorts of parts and moments thereof. Proper diagrams, then, to the extent that they are true (i.e. are such as to depict existent structures in reality) will depict facts involving ordinary objects of these and similar sorts. Whether, or to what extent, the language can be extended to cope also e.g. with microphysical structures is a question we here leave open.

A directly depicting language seeks high representational adequacy, even at the expense of low expressive adequacy. Thus our language will 
have no facility to represent propositional attitudes, it will have no probabilistic machinery, and it will tolerate no vagueness. Work in formal philosophy of the last 70 years or so has concentrated overwhelmingly on problems of the representation of knowledge, which is to say on the various ways in which partial and sometimes incorrect information is acquired, stored and processed. Here, in contrast, we are interested not in the peculiarities of episteme and doxa, but purely in the idea of a direct depiction of reality taken for its own sake, which is to say independently of any concern as to how such direct depiction may play a role in our common concerns with reality as knowing subjects.

Valuable groundwork in this respect is to be found in Gustav Bergmann's "Notes on Ontology" of 1981. Here Bergmann formulates four conditions on what he calls an 'improved language', conditions we here reproduce in somewhat simplified form:

(F1) With four exceptions, every primitive mark of the improved language stands for an existent.

(F2) Every well-formed 'string' of the improved language stands for a determinate, i.e., for either a thing or a complex or a class.

(F3) Every primitive standing for a thing and every well-formed string of the improved language stands for one and only one existent; and, with a single exception (relating to classes), conversely.

(F4) (a) There are in the improved language no complex characters such as, say, green-andsquare. In the improved language, therefore, there are, by (F1), (F2), no derived predicates, whether introduced by an abstraction operator or (as one says, metalinguistically) by definitions.

(b) There are by (F3) in the improved language none of the expressions Russell called incomplete symbols. To epitomize both (a) and (b) in a way that throws some light on the reasons for them, the improved language contains no abbreviations.

(c) In the improved language there are no variables. Any notation using them prevents one from arriving at an ontologically adequate assay of quantification.

$A d(\mathrm{~F} 1):$ The problem of depicting putative intentional facts such as are expressed, e.g., by sentences like 'John is thinking of a unicorn' is one central experimentum crucis for a directly depicting language. Accordingly the most important exception of the four mentioned by Bergmann in the first of his four conditions is the primitive mark 'M' (roughly: 'is thinking of'), which for Bergmann is an ingredient of the expression of the connection between a thought and its intention. $\mathbf{M}$, in Bergmann' s view, 'stands literally for nothing' (p. 140). The diagrammatic language to be presented below, in contrast, satisfies Bergmann's first condition with no exceptions at all. On the other hand, this language may 
be weaker than that projected by Bergmann, in that it is not capable of expressing facts of the sort Bergmann expresses using ' $\mathbf{M}$ ' (if indeed there are such facts).

$A d$ (F2): Bergmann, as he himself tells us, uses the word 'string' merely provisionally, since he had come to the view that no linear notation can accommodate an ontologically adequate assay even of linear order. As it happens he, too, suggests an Euler-type notation for his improved language, in which for example the exemplification of what he calls a 'character' (for example green) in an object is represented (roughly) by inscribing the name of the object in a circle and the name of the 'character' in the space between this circle and a larger concentric circle drawn around it. ${ }^{9}$

$A d$ (F3): We, too, shall accept that every (true) diagram of our language will stand for one and only one existent. On the other hand, there are for us many cases where a single existent admits of being truly diagrammed in a multiplicity of distinct ways. Perhaps we can say that Bergmann was able to take seriously the goal of one diagram per entity because entities, for him, are ultimately very simple indeed. Here, on the other hand, we are interested in establishing a diagrammatic language that is able to provide different snapshots of one and the same segment of reality as it were on different levels or with different finenesses of grain, so that different sorts of detail are brought into focus. (Compare the way in which incompatibilities arise among different Fregean analyses of linguistic objects into saturated and non-saturated parts.) The ontologist then has a means of representing certain non-depictable facts positively, by allowing metadiagrammatic assertions, not officially a part of the diagrammatic language as such. That is, he can express such facts by means of assertions about the diagrams themselves. Thus for example he can assert that two entities are identical by means of a statement like $\Theta=\Sigma$, where $\Theta$ and $\Sigma$ are diagrams of the entities in question. He might consider also for example a substitution law to the effect that, if ' $\Theta=\Sigma$ ' is true, and if ' $\Gamma(\Sigma / \Theta)$ ' is like ' $\Gamma$ ' except that ' $\Theta$ ' has been replaced by ' $\Sigma$ ' in one or more places, then from $\Gamma$ one may infer $\Gamma(\Sigma / \theta)$.

$A d$ (F4)(a): We, too, in what follows, shall reject all derived terms for what is general, whether introduced by abstraction or by definition.

$A d$ (F4)(b) We shall also allow no Russellian incomplete symbols. However, since we allow different (true) diagrams of one and the same existent, and since some of these diagrams will turn out to be simpler than others, our language does in this sense incorporate a facility for something 
like abbreviations. Abbreviations, for us, are possible because of our caveat to (F3); but still, we accept nothing like definite descriptions.

$A d$ (F4)(c) We agree with Bergmann also in the rejection of variables. Reality is determinate, so that any notation capable of directly depicting this reality must also be determinate. ${ }^{10} \mathrm{We}$ shall, though, allow metavariables which will enable us to make assertions about our diagrams themselves.

\section{THE OIL-PAINTING PRINCIPLE}

Bergmann's four conditions are interesting and important, but he and his followers have been blinded by the successes of Fregean logic to the extent that their conceptions of an ideal or improved language still reveal elements of compromise as between the logical and epistemological purposes of knowledge-representation and the ontological purposes of object-representation appropriate to a directly depicting language as here conceived. Above all, Bergmann and his followers, in neglecting the mereological constraints at the heart of the idea of a directly depicting language and in seeking to hold too closely to the paradigm of Fregean logic, did not see that the principal mark of a characteristic language is that it satisfies:

The Oil-Painting Principle (also called 'Degen's Law'): If diagram $\Theta$ is a well-formed part of diagram $\Gamma$, then if $\Gamma$ is true, so also is $\theta .{ }^{11}$

This, too, is a metadiagrammatical principle affirming that one diagram can be inferred from another. A formulation of the intuition underlying the principle - an intuition shamefaced in its naivety - might be: every part of a representative work of art is also representative. Clearly, in a language which satisfies a principle of this sort, there can be no disjunctive, negative or hypothetical diagrams. ${ }^{12}$ Already at this level, therefore, the purposes of logic and the purposes of direct depiction are alien to each other, a fact that was perhaps recognized by Wittgenstein in the Tractatus, e.g. in his drawing of the distinctions between Elementarsatz and Satz and between Sachverhalt and Tatsache. The relation of making true, here, holds exclusively between the Elementarsatz and the Sachverhalt: the truth of Sätze is an entirely derivative matter. If, now, we define logical atomism as a view to the effect that only that can serve to make a sentence or proposition true which can be depicted in a directly depicting language, 
then it would follow that there are, for the logical atomist - and as Wittgenstein correctly saw - no disjunctive, negative or hypothetical states of affairs.

Can we accept a converse of the oil-painting principle, to the effect that, if $\Theta$ and $\Gamma$ are any true diagrams, then there is a true diagram containing $\Theta$ and $\Gamma$ as parts? This would ensure that diagrams constitute at least an upper semi-lattice on the obvious mereological ordering, with unit something like a complete diagram of the world. Or can we accept an even stronger principle, amounting to the affirmation of a unity of reality of an almost Spinozistic sort, which would assert that there is some true connected diagram containing any $\Theta$ and $\Gamma$ as parts? Problems will arise for any such law in virtue of the fact that we could not, in general, read off from the antecedently given diagrams what the relevant containing diagram (least upper bound) would be. Thus we should have no effective way of setting such a law to work. This, though, may be merely an epistemological matter, of no ultimate significance for ontology.

On the other hand it seems clear, at least on our intuitive understanding of the ways in which the bits of worldly furniture are related, that we can on no account embrace a general intersection law which would allow us to infer, from any given pair of diagrams, to their intersection or meet. For consider some pair of diagrams depicting disjuncta (in the sense of entities that have no parts in common). The intersection of disjuncta is, by hypothesis, empty. Yet it seems clear that a directly depicting language can on no account allow what might be called an empty or null diagram, since such a diagram would, by definition, depict nothing at all. Thus the system of diagrams cannot constitute a lower semi-lattice. Mereology, too, allows nothing like a null element, and thus the mereological intuitions at the heart of the idea of direct depiction make themselves once more clearly felt.

\section{PRIMITIVES AND DEFINITIONS ${ }^{13}$}

The goal of a directly depicting language is that of constructing a system of diagrams that will allow the direct and adequate depiction of a maximum number of (kinds of) ontological facts. As we have seen, some sorts of facts - for example all facts involving negation or disjunction - cannot be depicted directly. Identity, too, is a relation of this sort. Bergmann gives arguments for supposing that diversity is a positive fact, ${ }^{14}$ and certainly some sorts of diversity (for example mereological discreteness) can be 
directly depicted. Facts of identity would then be negative facts, which would make it understandable that they cannot be directly depicted.

How a finally acceptable directly depicting language will look, will clearly depend on what the world is like. Hence the route one takes towards the construction of such a language will reflect one's initial ontological intuitions, which might for example be of a Fregean, Machian, Tractarian, Chisholmian, or Bergmannian sort. In any case, it will be necessary to start with some one or other candidate world-picture and to experiment with the construction of a directly depicting language appropriate thereto. Perhaps standard predicate logic is the best one can achieve in the direction of a directly depicting language for a universe enjoying a certain sort of settheoretic structure. The fact that standard predicate logic fails almost every test of direct depiction might then serve as a reason for abandoning set theory as a basis for ontology. In general, however, one's candidate world-picture will not, in this way, need to be abandoned entirely. Rather, it will have to be tentatively adjusted in light of problems encountered when the attempt is made to represent it in a diagrammatic way. Such adjustments may then lead in turn to new principles of direct depiction, and these may reflect back in the form of adjustments to the underlying ontology, in a cycle which may be repeated for as long as it takes to reach a match of language and ontology of the appropriate sort.

Before turning to the directly depicting language itself, then, we need to make clear the intitial repertoire of ontological categories and relations between categories to which the various bits and pieces of its machinery shall correspond, in order that the cycle of diagrammatic experimentation can begin. Here we shall follow Aristotle and some scholastics in taking as our initial focus the notion of an individual substance or continuant, examples of which would be such ordinary objects as human beings, oxen, logs of wood. Associated herewith is the Aristotelian category of individual accident (individual qualities, actions and passions, Rupert's present knowledge of Greek, an electric charge, a bruise, a blush). Accidents are said to 'inhere' in their substances, a notion which will be defined more precisely in what follows in terms of the concept of specific dependence. Substances and accidents together will constitute what we shall call the 'atoms' of our system of ontology.

'Individual', here, is a primitive term, and its precise meaning will become clear only in the course of what follows. As we understand the term, however, it is ruled out that there might be abstract individuals: to be individual is to be fully a part of the constantly changing order of space and 
lime. We do not, however, wish to presuppose that everything is individual: good candidate examples of non-individuals might be universals like red, entities capable of being exemplified by or realized in a multiplicity of individuals. Hence we shall recognize stocks of both individual and non-individual names, leaving open the possibility that the latter may turn out to be empty. Both individuals and non-individuals may be simple or complex. We adopt hereby a convention to the effect that a whole is individual if any part is individual (so that all the parts of a nonindividual are themselves non-individual). Moreover, we shall assume that non-individuals are such as to exist only in re, which is to say that they exist only insofar as they are exemplified by or realized in individuals. This implies the following:

Weak Law of Immanent Realism: If there is anything, then there is something individual of which it is a part.

We shall also assume, for present purposes, that no individual is such as to exist necessarily. From which it will follow that non-individuals, too, enjoy a merely contingent existence (they exist only for as long as, and to the extent that, there are individuals in which they are realized or exemplified.)

The notions now defined (in terms of the primitives 'individual', 'part', ${ }^{15}$ and 'is necessarily such that') will be the formal or categorial notions by which the further construction of our diagrammatic language will be motivated and in terms of which it will be described. $x$, y, etc., are metavariables standing in for proper names of individuals and nonindividuals. We define first of all:

$\mathrm{x}$ is disjoint from $\mathrm{y}=\mathrm{df}$. $\mathrm{x}$ and $\mathrm{y}$ have no parts in common.

$\mathrm{x}$ is discrete from $\mathrm{y}=\mathrm{df} . \mathrm{x}$ and $\mathrm{y}$ are individuals which have no individual parts in common.

Jules and Jim are discrete from each other in this sense. If, however, they contain as parts in common universals such as human or animate, then they are not disjoint.

To capture the notion of inherence, the relation holding between an accident and that which it is an accident of, we now put:

$\mathrm{x}$ is specifically dependent on $\mathrm{y}=\mathrm{df}$. (1) $\mathrm{x}$ is an individual, and (2) $x$ and $y$ are such as to have no individual parts in common, and (3) $\mathrm{x}$ is necessarily such that it cannot exist unless $\mathrm{y}$ exists. 
A headache, for example, is specifically dependent on me (as also on my head). This is a case of one-sided specific dependence (for it is clear that I am not specificially dependent on my headache). As we shall see, however, there are also cases where entities stand to each other in relations of mutual or reciprocal dependence.

We can now define:

$z$ is the mereological sum of $x$ and $y=d f$. (1) $x$ is part of $z$, and (2) $y$ is part of $z$, and (3) no part of $z$ is disjoint from both $x$ and $\mathrm{y}$.

$\mathrm{x}$ is a one-sidedly separable part of $\mathrm{y}=\mathrm{df}$. (1) $\mathrm{x}$ is a proper part of $y$, and (2) some part of $y$ discrete from $x$ is specifically dependent on $\mathrm{x}$, and (3) $\mathrm{x}$ is not specifically dependent on any part of $y$ discrete from $x$.

$\mathrm{x}$ is for example a thinker and $\mathrm{y}$ is the mereological sum of $\mathrm{x}$ together with some one of $x$ 's thoughts.

$x$ and $y$ are mutually separable parts of $z=d f$. (1) $z$ is the mereological sum of $x$ and $y$, and (2) $x$ and $y$ are discrete from each other, and (3) $x$ is not necessarily such that any individual part of $y$ exists and (4) $y$ is not necessarily such that any individual part of $\mathrm{x}$ exists.

$\mathrm{z}$ is, for example, a pair of stones, and $\mathrm{x}$ and $\mathrm{y}$ the stones themselves. It can be seen to follow from the definition that only individuals are candidates for being either one-sidedly or mutually separable parts.

$\mathrm{x}$ is atomic $=\mathrm{df}$. (1) $\mathrm{x}$ is an individual, and (2) no part of $\mathrm{x}$ has either one-sidedly or mutually separable parts.

If $\mathrm{x}$ is atomic, we can infer that all proper parts of $\mathrm{x}$ stand in mutual dependence relations to other proper parts of $x$. The concept of an atomic entity does not yet capture the concept of substance however. For what is atomic need not, according to this definition, be independent or selfsustaining. Thus there might be dependent entities, for example in the realm of qualities, which are atomic by our definition. Accordingly we define:

$\mathrm{x}$ is substantial $=\mathrm{df}$. (1) $\mathrm{x}$ is atomic and (2) $\mathrm{x}$ is not specifically dependent on any other entity. 
We are still not home, however, for our definition of 'substantial' is sillisfied by quantitative parts of substances, such as Darius's (undetached) arm. The latter is atomic, because any proper part must share a boundary with some adjacent part and this would imply, as we shall see, a (mediate) dependence on the latter. But the undetached arm (or a growing tree) is clearly not a substance, either; for it, too, shares a houndary with some other entity, and is therefore once more dependent on something other than itself. What we loosely refer to as Darius's arm becomes a substance only on becoming detached, when it acquires a houndary of its own. Much of this will become clearer when once the relevant portions of the directly depicting language have been set down. lior the moment we simply note that in order to arrive at a definition of substance we shall need to take account of the notion of boundary. To this end we introduce a new sort of dependence:

$\mathrm{x}$ is boundary dependent on $\mathrm{y}=\mathrm{df}(1) \mathrm{x}$ is a proper part of $\mathrm{y}$, and (2) $\mathrm{x}$ is necessarily such that either $\mathrm{y}$ exists or there exists some part of y properly including $x$, and (3) each individual part of $\mathrm{x}$ satisfies (2).

Thus for example the boundary of a billiard ball is a part of and is boundary dependent on the ball itself. We can now define:

$\mathrm{x}$ is self-boundingly substantial $=\mathrm{df}$. (1) $\mathrm{x}$ is substantial, and

(2) there is no $y$ that is boundary dependent on $x$ and on some object discrete from $x$.

That Darius's undetached arm (or, again, a tree that is growing out of the ground) do not satisfy this definition will allow us to define substances as minimal self-boundingly substantial objects:

$\mathrm{x}$ is a substance $=\mathrm{df}$. (1) $\mathrm{x}$ is self-boundingly substantial and

(2) no proper part of $x$ is self-boundingly substantial.

Parallel to the distinction between 'substantial' and 'substance', we now have a distinction between 'accidental' and 'accident':

$\mathrm{x}$ is an accidental of $\mathrm{y}=\mathrm{df}$. (1) $\mathrm{x}$ is atomic and (2) $\mathrm{x}$ is not substantial and (3) $\mathrm{x}$ is specifically dependent on $\mathrm{y}$.

This definition has the useful property that it allows us to recognize that there are accident-like entities which relate to undetached or quantitative parts of substances as accidents proper relate to substances themselves. 
(Consider the individual redness of the snake that is half red and half white.)

In what follows our attentions will be directed principally to accidents of substances. Hence we shall define:

$\mathrm{x}$ is an accident $=\mathrm{df}$. $\mathrm{x}$ is an accidental of a substance.

We can now define:

$\mathrm{x}$ is an atom $=\mathrm{df} . \mathrm{x}$ is either a substance or an accident.

The atoms are, as it were, the most privileged, most natural, most selfcontained and well-rounded examples among the domain of atomic entities in general.

What is substantial is always part of some substance, and what is accidental is always part of some accident. From this it follows that to recognize the categories of substantials and accidentals is to add nothing new to the totality of what exists. It reflects cuts skew to those which pick out substances and accidents, and the latter, we suggest, reflect the most natural joints in reality. In what follows, accordingly, we shall ignore what is substantial as such, and see individual reality as being divided into substances and accidents alone. The world, then, is the totality of atoms, and the relation of specific dependence is the bond which holds these atoms together in molecules of different sorts.

To capture the latter notion we first of all define:

$\mathrm{x}$ is closed under specific dependence $=\mathrm{df}$. no part of $\mathrm{x}$ is specifically dependent on any entity discrete from $\mathrm{x}$.

(Everything substantial is closed under specific dependence in this sense.)

We can now say:

$\mathrm{x}$ is a molecule $=\mathrm{df}$. (1) $\mathrm{x}$ is closed under specific dependence, (2) $x$ contains a substance as a proper part, and (3) any pair of discrete parts of $\mathrm{x}$ are connected, directly or indirectly, by relations of specific dependence.

If, as seems reasonable, we exclude the possibility of what we might call lazy atoms, which is to say atoms which do not enter with other atoms into molecules of any sort, ${ }^{16}$ then it would follow that the world is not only the totality of atoms; it is also the totality of molecules. Again, no contradiction arises here, since the two given assays of the totality of what exists reflect cuts at different levels. Unlike Wittgenstein (and Aristotle, and 
Bergmann) we are not disturbed by the possibility of ontological inventories which reflect different sorts of modes or thicknesses of division in this way. Already every extended thing can be seen as being sliced along an infinity of different internal boundaries. This possibility will, however, imply that the idea of a single universal diagram is an idea that must be treated with caution.

Substances, as we have seen, may have substantials as proper parts. Accidents, correspondingly, may have accidentals as proper parts. Both substances and accidents may also, however, have certain essential parts, parts whose destruction leads to the destruction of the whole. Jim's individual humanity may be counted as an essential part of the substance Jim in this sense. Hue, saturation and brightness may similarly be taken as essential parts of that accident which is Jim's whiteness.

To capture this notion of essential part we shall introduce the terminology of 'sub-atoms'.

$\mathrm{x}$ is a sub-atom $=\mathrm{df}$. (1) $\mathrm{x}$ is a proper part of an atom and (2) $\mathrm{x}$ is not substantial or accidental.

Further examples of such essential parts are: the individual shape or mass of a body, the individual pitch, timbre or loudness of a tone. (If we entirely deprive a tone of its loudness we thereby destroy the tone itself.) As in the case of the hue, saturation and brightnesss of a colour, so also here, we are dealing with a case of three-fold mutual dependence. Indeed it turns out that many varieties of sub-atoms are entities which cannot as a matter of necessity exist except in consort with other sub-atoms of specific sorts.

Accidents may be relational or non-relational. They are relational if they depend upon a plurality of substances. Non-relational accidents are attached, as it were, to a single carrier, and thereby form, with their respective substances, the simplest kind of molecular whole. Relational accidents join a plurality of carrier-atoms together into more complex molecular wholes. Two or more individuals (be they atoms, sub-atoms or molecules) not joined together either by bonds or by further relational accidents constitute what we might call an aggregate:

$\mathrm{x}$ is an aggregate $=\mathrm{df}$. $\mathrm{x}$ contains a pair of discrete parts that are not connected, mediately or immediately, by relations of specific dependence. 
Our job, now, is to construct a diagrammatic language capable of representing perspicuously the above-mentioned categories of entities.

Substances are independent atoms, accidents are dependent atoms. An independent atom is an entity that is in need of no other individual entity outside itself in order to exist. Examples of substances might be: a thinker, a stone, a separated twist of DNA (if such there be). ${ }^{17}$ Examples of substantial entities which are not substances might be: a hemisphere of a planet, a twist of DNA in Chisholm's leg.

To depict substances we shall employ solid frames, which are to be understood in some respects after the manner of the ovoids of Euler or Venn diagrams. ${ }^{18}$ Thus:

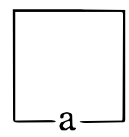

shall depict the independent atom whose proper name is a. We shall follow the convention that a proper name refers to the entire contents of the frame upon which it is inscribed. We shall adopt the (broadly Wittgensteinian) convention that all entities (whether individual or not) have a unique proper name and all proper names denote unique entities. Unlike Wittgenstein however we shall allow that proper names may depict parts of what other proper names depict. Thus for example

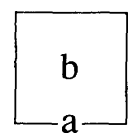

shall signify that $b$ is a part of the independent atom a. (The diagram does not tell us whether $b$ is or is not an atom, i.e. it does not tell us to what formal category the individual b belongs. Nor, incidentally, does it tell us whether $b$ is a proper or improper part of the substance a.)

Because no substance is ever a proper part of another, it follows that

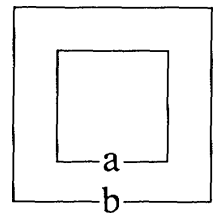


is always false. ${ }^{19}$ Here we accept Aristotle's view: a substance does not hilve any substances as proper parts (though of course every substance (anl, in different ways, be dismembered into substances - for example into :rrms and legs, or conceivably also into separate molecules).

Given our definitions, it will turn out that

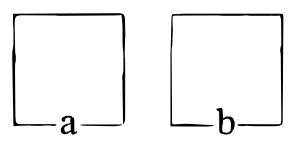

which signifies that independent atom a is discrete from independent at om $b$, is in contrast always true.

If one substance could overlap with another in the sense of sharing some individual part in common, without either including or being included in this other, then this state of affairs would be represented by:

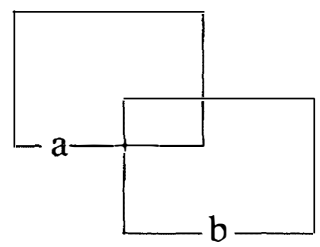

Siamese twins do not constitute an example of a structure such as this, since neither twin is, before separation, a substance. Touching billiard balls would not constitute an example, either, since the portion of boundary they share is not an individual, on our account.

Degen's law allows us to infer from

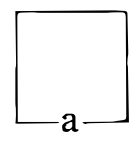

to:

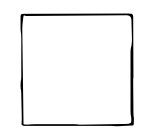

The latter may for the moment be read as signifying that some independent atom exists. ${ }^{20}$ 
From

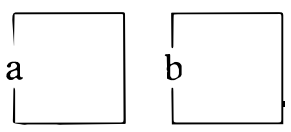

we may infer:
a
b

The latter is, be it noted, a fact in the sense of TLP 3.142: it is not a list, or set, of names.

From

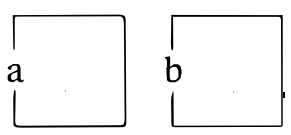

we may also however infer:

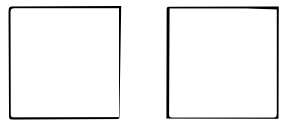

Read: 'two independent atoms exist.'

The metadiagrammatic:

$$
a=\square
$$

which comes close to saying what Wittgenstein says cannot be said at 4.126 , is either necessarily true or necessarily false, according to whether $\mathrm{a}$ is or is not a substance. 
We also have the inference:

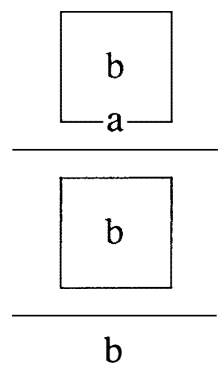

i.e. from $b$ is a part of independent atom a we may infer: $b$ is a part of some independent atom, and from this we may infer: $b$ exists.

Note that from:
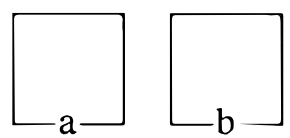

we cannot infer that $\mathrm{a}$ and $\mathrm{b}$ are disconnected, i.e. that they are not linked via relations of dependence through the mediation of other entities. The fact that an entity is a mere aggregate in this sense is a negative fact and therefore (as we have seen) not something that can be directly depicted. The property of being an aggregate is not diagrammatically perspicuous: not everything that is depicted by a non-connected diagram is itself nonconnected in reality.

\section{ACCIDENTS}

An accident or dependent atom a is an entity which is necessarily such that it requires some other discrete entity or entities in order to exist but not vice versa. The required entities (here substances) are called the carriers or termini or fundamenta of the dependent atom.

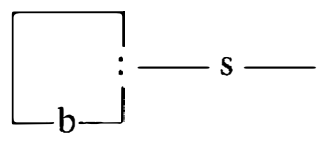

shall depict a dependent atom $b$ with one terminus. 


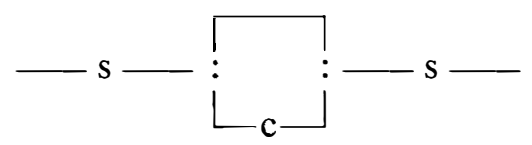

shall depict a dependent relational atom $\mathrm{c}$ with two termini, etc.

The ' $s$ ' inscribed on the links connecting dependent frames to their carriers here signifies the relation of specific dependence.

Thus a picture of a dependent atom comprises:

(1) a frame with one or more perforated walls from each of which protrudes (2) a dependence link representing the specific dependence of the dependent atom upon the relevant carrier or fundament.

(1) and (2), here, are syncategorematic parts of the diagrams in which they occur. That is to say, neither is well-formed (categorematic) for the purposes of Degen's law inferences.

The diagrammatic equivalent of the notion of closure introduced above is the concept of a closed diagram, i.e. a diagram all of whose links are connected to the walls of other frames. This suggests the following

Closure Law: If $\theta$ is a diagram in which dependent atoms or sub-atoms are depicted, then there is some unique smallest diagram $\operatorname{cl}(\theta)$, the closure of $\theta$, in which all the relevant containing atoms and independent carrier-atoms are depicted. ${ }^{21}$

It is possible that dependent atoms may themselves serve as carriers for further dependent accident-like entities of a higher order. For example the individual redness of my bruise is dependent on the bruise itself, which is in turn dependent on me. Such chains of one-sided dependence-relations must however come to an end after a finite number of steps. Dependent atoms never occur alone, but are in every case constituents of molecules in which their carriers are also contained. Thus we can embrace the following:

Principle of Ontological Well-Foundedness (ontologische Begründungsaxiom): That on which a dependent atom depends is always such as to include one or more independent atoms as parts.

This may be also be formulated as the: 
Strong Law of Immanent Realism: If there is anything, then there is a substance.

We would more precisely need to affirm a principle to the effect that there are a finite number of dependence steps between dependent atom and independent carrier - a principle to the effect that, leaving aside the mutual dependence of sub-atoms, every dependence diagram is a finite non-cyclical graph.

The principle of well-foundedness does not, however, allow us to infer c.g. from

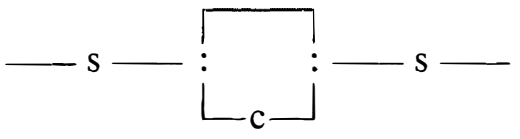

10:

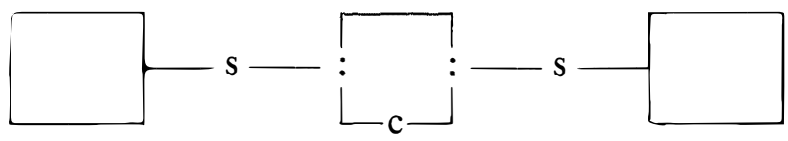

since we also have cases such as the following, where a relational accident involves only a single substance:

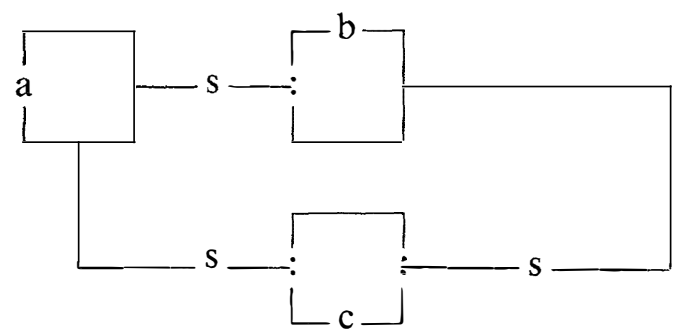

Here $\mathrm{a}$ is (for example) a thinker, $\mathrm{b}$ an act of seeing, $\mathrm{c}$ a state of enjoying b. (Aristotle, incidentally, affirms that accidents of accidents are always also accidents of substances in this sense. ${ }^{22}$ ) 


\section{SUB-ATOMS (MUTUALLY DEPENDENT PARTS OFATOMS)}

We have distinguished between essential parts of a substance, for example Rupert's being heavy or having a shape, and accidents of a substance, for example Rupert's present headache, or present jump. And we have drawn a similar distinction also in relation to accidents, for example between the brightness of a colour, on the one hand, and the noise of a walk, on the other. (No colour can exist without some brightness; a walk can however exist without it being the case that there is an accident of noise inhering in it.) It is for the moment of no consequence that the line between the two sorts of individual property may be somewhat difficult to draw. Our initial concern is merely to construct a directly depicting language that is able to cope with both sorts of case.

We shall assume in what follows that sub-atoms always stand in mutual dependence relations to each other. A picture of a sub-atom shall therefore consist of:

(1) a frame with one or more broken walls from each of which protrudes

(2) a dependence arrow representing the dependence of the dependent atom upon some one or more other sub-atoms and upon which is inscribed an ' $s$ ' or ' $b$ ', indicating that the dependence involved is specific or boundary dependence, respectively. ${ }^{23}$

In the simplest case, sub-atoms stand in relations of pair-wise mutual specific dependence, for example as follows:

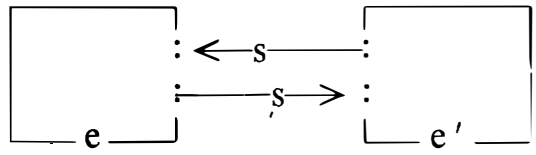

Clearly, similar diagrams can be constructed where n-fold mutual dependence obtains, and the idea is that the internal structure of every atom could be represented exhaustively by a family of complex diagrams of the given form, representing cuts of different sorts and in which all subatoms are eventually displayed.

The arrow here indicates direction of dependence and serves to distinguish mutual dependence from the one-sided dependence involved in the case of dependent atoms. In all closed diagrams, each dependence 
:1rrow connects its frame to the wall of some other frame. A picture of a sub-atom is further characterized by the fact that in a closed diagram it is reciprocally connected by dependence arrows to other frames.

From

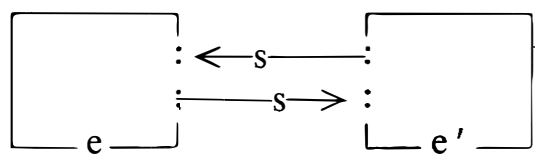

we may infer

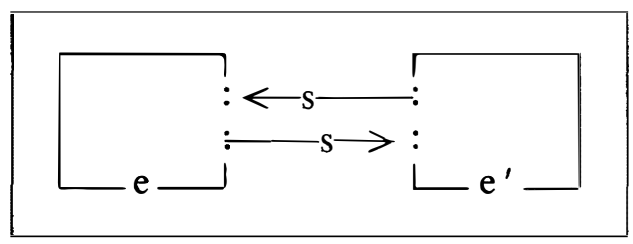

For if e and e' really do depend upon nothing other than themselves in order to exist, then together they must constitute an independent atom.

\section{BOUNDARIES AND BOUNDARY DEPENDENCE}

We are supposing, with Aristotle (though in a different terminology), that Darius is an independent atom. Then by definition of atom, no part of Darius is able to exist except in association with all the other parts. Let ' $a$ ' signify Darius's arm, as it is, now, attached to the remainder (the torso) $t$ of Darius. Then there is some boundary c running between a and $t$, and inspection reveals that $\mathrm{c}$ is boundary dependent on both a and $\mathrm{t}$, by our definition above. In particular, $\mathrm{c}$ is a part of both a and t. Boundaries are in this sense - and like universals - multiply located.${ }^{24}$ Moreover, both a and $t$ are specifically dependent upon c. For a and $t$ are in fact defined and delineated only via c. Any alternative delineation would capture not a and $\mathrm{t}$ but more or less distant cousins. a and $\mathrm{t}$, then, exist as a matter of necessity only if $c$ exists. But this is just the definition of specific dependence. The whole of Darius, on this particular parsing of his structure, may accordingly be represented thus: 


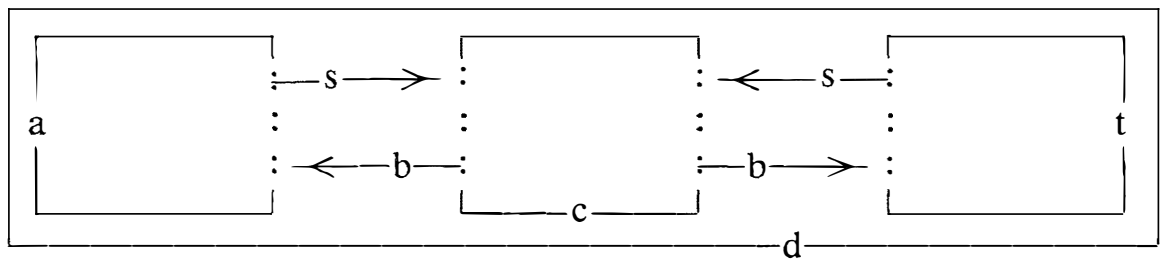

Note that this representation is in order as it stands, in spite of the fact that $\mathrm{c}$ is part of both a and $\mathrm{t}$, because it is not an individual part.

How, now, does Darius's arm a ' relate to Darius, when once it has been actually removed from Darius's torso? Precisely thus:
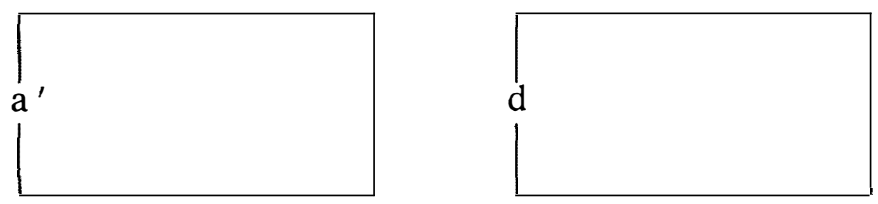

Note that we have here identity of Darius himself across the temporal interval during which the scission takes place. Darius, as a substance, is self-identical from the beginning to the end of his existence. But neither before nor after the removal of his arm is Darius identical with any substantial part of himself (something which follows trivially from the definition of substance and from our assumption that Darius is such as to satisfy this definition). After the operation Darius (now minus arm) is still an atom in his own right. The two diagrams allow us to see very clearly why this is so. $\mathrm{a}$ and $\mathrm{a}^{\prime}$ are non-identical, because $\mathrm{a}^{\prime}$ is a substance and a is merely substantial. No such difference of category arises in the case of Darius before and after the loss of his arm.

Boundaries correspond to possible cuts or parsings through reality. All extended objects allow an indefinite number of cuts or parsings of this sort, which are typically skew to each other. This possibility is, for Brentano, a mark of what is continuously extended. ${ }^{25}$ Skew to all these parsings, however, are divisions, e.g., into sub-atoms. 
Return, now, to the diagram:

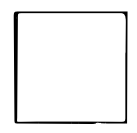

for which we suggested the reading 'some independent atom exists'. Can this diagram truly be counted as satisfying the conditions on a directly depicting language? To see why there is a problem here, consider the following experiment. Imagine a pair of distinct diagrams, say

and
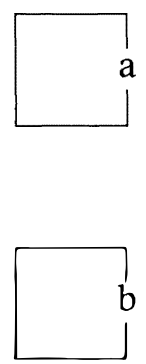

inscribed on separate sheets of paper. Suppose, further, that both diagrams are true, and in such a way that they depict discrete entities which we might assume to be several miles apart. Imagine, now, that Degen's law is applied to each diagram, so that on our separate sheets of paper we now have:
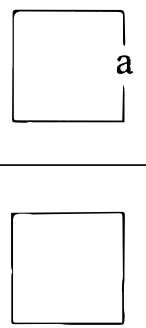
and:

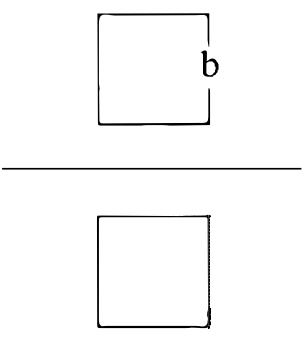

Do the two resulting diagrams, now, depict identicals? This seems counterintuitive, given that the initial diagrams depicted discrete and separate entities, and that the two resulting diagrams are such as to depict parts of these entities. On the other hand it seems that some, at least, of our (diagrammatic) intuitions point in the opposite direction. These intuitions, which amount to the thesis that there can be no ambiguous diagrams - or as we expressed it in our treatment of Bergmann, above: every (true) diagram stands for one and only one existent - can be packaged together as the:

Principle of Diagrammatic Rigidity: If $\theta$ depicts $x$, then there must be something in $\theta$ in virtue of which it does so. ${ }^{26}$

It follows from this principle that (true) equiform diagrams picture one and the same entity. But what can this entity be, in the case of diagrams like:

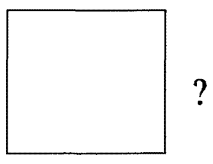

The only candidate which here presents itself would seem to be the formal universal substance, an entity which would serve as a non-individual part of every individual substance. If this is correct, then the diagram in question can be read as signifying either:

Some individual substance exists,

or:

The universal substance exists (is realized, is exemplified). 
This argument (or ontological experiment) is, I believe, sound and yields the conclusion that certain formal universals exist. Moreover, it can be extended in such as way as to lend credence also to the thesis that there are material universals such as the humanitas of Rupert, or the redness that is shared in common by Rudolf's nose and this telephone box. The diagrammatic language can, accordingly, be extended to depict also material universals in this sense. Some classical positions as to the nature of universals then fall out as incapable of realization within the framework of a universal characteristic. Other such positions, however, can be so realized, in ways which yield alternative directly depicting languages of surprising force. The issue as to which of these alternatives comes closest to depicting the joints of reality remains, however, open.

\section{NOTES}

' Peirce 1933, 4.530. All references to Peirce in this section are taken from this passage.

2 See Descartes' letter to Mersenne of 20 November 1629, Engl. trans. in Descartes, Philosophical Letters, A. Kenny, ed., Oxford: Blackwell, 1970, p. 6.

3 Meyer 1957, p. 57.

${ }^{4}$ Quoted in Brentano 1982, pp. x-xi.

${ }^{5}$ Dummett 1981, p. 482.

${ }^{6}$ We recall that Gauss, too, conceived algebra as a 'science of the eye', a matter of observation, though of objects of a highly recondite character: cf. Peirce 1.34, 4.233.

7 Cf. Peirce, 4.356.

${ }^{8}$ See e.g. Aristotle, Met., 1027 b 22, 1051 b 32ff.

"See p. 149 of "Notes on Ontology".

${ }^{10}$ Kit Fine's 1985 rests essentially on a rejection of this idea, and makes possible for the first time a coherent formulation of an ontology in relation to which variables might properly be exploited in a directly depicting language.

${ }^{11}$ See Degen 1978, Smith and Mulligan 1982, pp. 81-91.

12 Thus in particular Bergmann's notation of concentric circles cannot serve as the basis of a directly depicting language, since it allows disjunction to be treated as just another character (1981, p. 150).

${ }^{13}$ Much of this section bears a strong indebtedness to Chisholm's forthcoming work on the theory of categories. See also his 1982a, 1982b, 1984.

${ }_{14} 1981$, p. 145.

15 'Part', here, means always 'proper or improper part'.

${ }^{16}$ Cf. Tractatus, 3.328, 5.47321.

${ }_{17}$ In some ontologies the atoms are constituted not by substances (ordinary objects) but by other sorts of entities. The differences here may be material - as in the case where one takes sense data as atoms. Or they may be formal: thus Sachverhalte, on one reading of the Tractatus, take the place of our atoms, and Wittgensteinian simple objects take the place of our sub-atoms. 
18 See Smith and Mulligan 1982 and 1983 for more details of this aspect of the theory.

19 'Always' in the sense that it is false for every pair of proper names ' $a$ ' and ' $b$ '. (Empty proper names have, it will be remembered, been excluded from consideration.)

${ }^{20}$ It is something like a prototype in the sense of Tractatus, 3.333.

${ }^{21}$ It is worth noting that ' $\mathrm{cl}$ ( )' here satisfies the usual Kuratowski axioms for a topological space. It is worth noting also that, like the converse Degen laws considered above, the closure law is not effective in the sense that we are not, in general, able to construct the diagram whose existence the law would purport to guarantee.

22 For a discussion of this law in Aristotle and Brentano see my 1987.

${ }^{2.3}$ Again, neither (1) nor (2) is when taken alone well-formed for the purposes of Degen's law inferences.

${ }^{24}$ There is, in fact, as Brentano acknowledged (1976), a deep and surprising parallel between boundaries and universals, so that much of what is necessary for an adequate diagrammatic treatment of the latter can be derived from the treatment of the former.

25 See, again, his 1976.

${ }^{26}$ Cf. also Bergmann's 'fundamental principle of ontology': different complexes must differ in a constituent. (Cf. his 1967, p. 22.)

Note that the principle given in the text runs counter, e.g. to Wittgenstein's idea that the depiction of an object or complex might be catered for in part psychologically, in terms of some extra-diagrammatic 'projection', at 3.11-13. The psychological connotations are incidentally no longer present at 4.0141 .

\section{REFERENCES}

Bergmann, Gustav 1967 Realism. A Critique of Brentano and Meinong, Madison: University of Wisconsin Press. , 1981 “Notes on Ontology", Noûs, 15, 131-64.

Brentano, Franz 1976 Philosophische Untersuchungen zu Raum, Zeit und Kontinuum, ed. by R. M. Chisholm and S. Körner, Hamburg: Meiner, Eng. trans., Philosophical Investigations on Space, Time and the Continuum, by B. Smith, London: Croom Helm, 1988. 1982 Deskriptive Psychologie, R. M. Chisholm and W. Baumgartner, eds., Hamburg: Meiner.

Chisholm, R. M. 1982 Brentano and Meinong Studies, Amsterdam: Rodopi. , 1982a "Beginnings and Endings", in Chisholm 1982, 114-24. 1982b "Brentano's Theory of Substance and Accident", in Chisholm 1982, 3-16. 1984 “Boundaries as Dependent Particulars", Grazer Philosophische Studien, 10, 87-95.

Degen, Wolfgang 1978 “Sketch of a Rational Grammar”, unpublished MS., University of Erlangen.

Dummett, M. A. E. 1981 The Interpretation of Frege's Philosophy, London: Duckworth. Fine, K. 1985 Reasoning with Arbitrary Objects, Oxford: Blackwell.

Grossmann, R. 1983 The Categorial Structure of the World, Bloomington: Indiana University Press.

Husserl, E. 1900/01 Logische Untersuchungen, 1st ed., Halle: Niemeyer, 2nd ed., 1913/21; Eng. trans. by J.N. Findlay, Logical Investigations, London: Routledge and Kegan Paul, 1970. 
Meyer, R. W. 1957 “Joachim Jungius und die Philosophie seiner Zeit” in Die Entfaltung der Wissenschaft, Hamburg: J. J. Augustin.

Peirce, C. S. 1933 Collected Papers, Cambridge, Mass.: Harvard University Press.

Smith, B. 1987 "The Substance of Brentano's Ontology", Topoi, 6/1, 39-49.

Smith, B., ed. 1982 Parts and Moments. Studies in Logic and Formal Ontology, Munich: Philosophia.

Smith, B. and Mulligan, K. 1982 "Pieces of a Theory", in Smith, ed., 15-109. , 1983 "Framework for Formal Ontology", Topoi, 3, 73-85. 